\title{
Recomendaciones para la realización de traqueostomía y manejo de paciente traqueostomizado luego de diez meses de pandemia COVID-19: revisión de la literatura
}

\author{
Recommendations for performing a tracheostomy and management \\ of a tracheostomized patient after ten months of COVID-19 pandemic: \\ a review of the literature
}

José Ignacio Rojas B. ${ }^{1,2}$, Nicolás Utrera Q. ${ }^{1}$, Cristián Papuzinski A. ${ }^{1-4}$

\section{Resumen}

La pandemia causada por el coronavirus designado SARS-CoV-2 ha tenido una propagación y efectos a nivel global importantes, generando desafíos para los distintos equipos médicos. Los otorrinolaringólogos se encuentran en particular riesgo por su manejo de la vía aéreodigestiva en la mayoría de pacientes y procedimientos. Dentro de éstos, la traqueostomía se observa como uno de aquellos procedimientos de alto riesgo que se verán involucrados en el manejo de pacientes con patología COVID-19 confirmada o posible. Es por ello que se deben tomar una serie de consideraciones para el correcto manejo, con el objetivo de disminuir los riesgos de contagio. En esta revisión se resumen las principales recomendaciones descritas por distintas sociedades de otorrinolaringología y cirugía de cabeza y cuello del mundo, así como de trabajos publicados relacionados. En resumen, se define un plan preoperatorio que considere el status infeccioso del paciente y elección cuidadosa por equipo multidisciplinario. Para la cirugía destaca utilizar elementos de protección personal disponibles, considerar las condiciones de la sala operatoria, equipo quirúrgico limitado y más experto. Durante el procedimiento se recomiendan una serie de medidas para disminuir el riesgo de exposición de aerosoles y secreciones. El manejo posoperatorio debe considerar cuidados especiales para el manejo de la cánula y sistema de ventilación.

Palabras clave: COVID-19, traqueostomía, recomendaciones, riesgo, aerosoles.

\begin{abstract}
The pandemic caused by the novel coronavirus designed SARS-CoV-2 has rapidly spread across the world, challenging all medical teams. Otolaryngologists are at particular risk because of interventions and care of aerodigestive secretions. At this level, tracheostomy it seen as a highly risk procedure involved in COVID-19 infected or suspected patients. Therefore, its important to take considerations for the correct care of these patients to decrease the potential contagious risk. In this revision we summarize the principal recommendations published by the main societies of otolaryngology and head and neck surgery among the world, as well as related published papers. In summary, a preoperative plan is performed, knowing the infectious status and a careful selection of patients by a multidisciplinary team. For the surgery, it is strongly recommended to wear personal protective equipment available, select the best conditions for the operating room, limited and most experimented surgery team. During the surgery, its recommended to take care for minimize the time of exposure to aerosolized secretions. Postoperative care should consider special measurements for cannula and ventilation systems.
\end{abstract}

Keywords: COVID-19, tracheostomy, recommendations, risk, aerosol.
${ }^{1}$ Departamento de Especialidades, Cátedra de Otorrinolaringología, Escuela de Medicina, Facultad de Medicina, Universidad de Valparaíso. Viña del Mar, Chile.

${ }^{2}$ Servicio de

Otorrinolaringología, Hospital Carlos Van Buren. Valparaíso, Chile.

${ }^{3}$ Centro Interdisciplinario de Estudios en Salud (CIESAL), Universidad de Valparaíso. Valparaíso, Chile. ${ }^{4}$ Departamento de Cirugía, Facultat dee Medicina i Odontologia, Universitat de València. València, España.

Los autores declaran no tener conflictos de interés.

Recibido el 9 de octubre de 2020. Aceptado el 15 de enero de 2021

Correspondencia: José Ignacio Rojas B. 3 poniente 220, depto. 1102 . Viña del Mar, Chile. Email: jose.rojasbr@postgrado. uv.cl 


\section{Introducción}

En diciembre de 2019, autoridades sanitarias de distintos establecimientos de salud en Wuhan, China, alertaron de brotes de neumonía de origen desconocido, epidemiológicamente ligados a un comercio de productos del mar de la zona․ El agente etiológico fue posteriormente identificado y designado como $S A R S-C o V-2^{2,3}$, convirtiéndose en el tercer coronavirus en generar un brote epidémico en humanos, luego del SARS-CoV en 2002 y del MERS-CoV en 2012 ${ }^{4,5}$. La OMS al 30 de septiembre ha reportado 33.502 .430 casos confirmados y 1.004.421 muertes a nivel mundial ${ }^{6}$, siendo Chile parte de estos casos, alcanzando a la misma fecha un total de 462.991 casos confirmados y 12.741 muertos $^{7}$. La transmisibilidad del virus entre humanos es a través de gotitas ${ }^{3}$ o por contacto directo, siendo contagioso desde períodos asintomáticos ${ }^{8}$, destacando además que puede permanecer viable y contagioso en aerosoles por horas y desde superficies inclusive durante días ${ }^{9}$. Una vez tenido contacto con el virus, su período de incubación varía entre 3-7 días, con un máximo de 14 días ${ }^{3,8}$. Si bien personas de todas las edades son susceptibles de contraer la enfermedad ${ }^{3}$, la mayor parte de los casos ocurren en personas de mayor edad, con una media de 59 años $^{10}$. Clínicamente los síntomas y signos más frecuentes son tos seca, fiebre, disnea y mialgias ${ }^{2,11}$, otros menos frecuentes son cefalea, hiposmia, diarrea, hemoptisis, tos productiva y rinorrea ${ }^{12}$. El diagnóstico debe ser confirmado por técnicas moleculares específicas (RT-PCR) ${ }^{8,12}$.

Dada la transmisión por medio de secreciones respiratorias, el personal de salud que maneje pacientes con patología de la vía aereodigestiva son los más susceptibles a infectarse ${ }^{13,14}$. En 2002 durante la epidemia del $S A R S-C o V$, la realización de traqueostomías correspondió al procedimiento quirúrgico más frecuentemente realizado ${ }^{15}$, el cual es considerado de muy alto riesgo de contagio dada la generación de aerosoles ${ }^{16}$, situando a otorrinolaringólogos, anestesistas y médicos de cuidados intensivos, en una situación de alta probabilidad de contraer esta enfermedad ${ }^{17}$.

Una revisión de 3 series de casos realizada por Tay y colaboradores en cuanto al manejo de traqueostomías quirúrgicas durante el brote en 2002, recopila los antecedentes que son la base del manejo durante la actual pandemia ${ }^{15}$. Los elementos de protección personal (EPP) son descritos como esenciales en el manejo y procedimientos efectuados en pacientes infectados, destacando, además, la necesidad de entrenamiento en las secuencias de colocación y retiro adecuado de los mismos. Con las medidas descritas por Tay, todo el personal participante de las cirugías se mantuvo sano durante el brote de SARS-CoV en $2002^{15,18}$.

La localización donde se realiza el procedimiento también resultó ser un tópico importante en el 2002, siendo de preferencia en la unidad de cuidados intensivos (UCI), en la cama del paciente, en habitaciones con presión negativa, la mayoría asociada a una ante-cáma$\mathrm{ra}^{18}$. En caso de realizarla en pabellón, también se recomendaron salas con presión negativa ${ }^{18}$.

El tiempo de exposición a secreciones aerosolizadas en el intraoperatorio se disminuyó asegurando una completa parálisis del paciente para evitar la tos, la realización del procedimiento por el equipo más experimentado, detención de la ventilación mecánica previo a la exposición traqueal y disminución del uso de aspiración durante la cirugía, o uso de sistemas cerrados con filtro vírico. Se prefirió la realización de la técnica quirúrgica sobre la percutánea, considerando más riesgosa esta última por mayor riesgo de manipulación de la vía aérea ${ }^{19,20}$. Por último, se hace énfasis a la descontaminación del equipo, la sala y correcta manipulación de material contaminado una vez finalizado el procedimiento ${ }^{15}$.

El realizar una traqueostomía involucra un gran desafío, especialmente en aquellos pacientes con sospecha o confirmación de infección por $S A R S-C o V$-2, es por ello que se hace necesario resumir la evidencia de las recomendaciones nacionales e internacionales de realización y manejo de traqueostomía luego de seis meses de la pandemia por COVID-19.

Se realizó búsqueda sistemática de la literatura en PubMed, EMBASE, Cochrane Central y Clinical Key, utilizando los términos Otolaryngology, ENT, SARS, SARS-2, coronavirus, COVID-19 and tracheostomy, tracheotomy. Además, se revisaron los sitios oficiales de las sociedades y asociaciones de otorrinolaringología y cirugía de cabeza y cuello. Se analizaron $\mathrm{y}$ resumieron todas las recomendaciones al 
realizar una traqueostomía y los cuidados posteriores que se deben tener en el contexto de la pandemia actual.

\section{Recomendaciones}

Se encontraron 876 artículos en la búsqueda inicial, seleccionando 55 tras la revisión de título y abstract para revisión de full text. Finalmente se incluyeron 38 artículos para la presente revisión. Se excluyeron aquellos no relacionados al procedimiento quirúrgico a revisar (15) y los duplicados (2). En la búsqueda manual de sociedades médicas, se seleccionaron 13 guías de 9 países: Estados Unidos $(2)^{21-23}$, España $(1)^{24}$, Reino Unido (3) $)^{25-27}$, Canadá $(1)^{28,29}$, Argentina $(1)^{30}$, Chile $(1)^{31}$, Francia $(1)^{32}$, Sudáfrica $(1)^{33} y$ Australia $(1)^{34}$. Las principales recomendaciones se resumen en la Tabla 1.

\section{Generalidades}

Todo procedimiento que tenga potencial de aerosolizar secreciones aerodigestivas en un paciente COVID-19 positivo (nasofibroscopía, traqueostomía, intubación orotraqueal, ventilación mecánica, cirugía endoscópica nasal entre otras), aumentan el riesgo de infección, y debieran ser evitadas o utilizadas sólo en caso estrictamente necesario ${ }^{13}$. The National Tracheostomy Safety Project (NTSP UK), reporta que existirían ciertos beneficios de manejo de pacientes traqueostomizados. Destaca la oportunidad de ofrecer un sistema sellado de soporte ventilatorio y un paciente con menos o sin sedación, lo que beneficiaría con menor tiempo y requerimientos de cuidados intensivos. No obstante, menciona las complicaciones de manejar a un paciente vigil no cooperador, además de la necesidad de un equipo médico con conocimiento en manejo de traqueostomías ${ }^{25}$.

\section{Elementos de protección personal}

Todas las sociedades científicas recomiendan el uso de EPP adicionales, más allá de los estándares, para realizar el procedimiento ${ }^{21-34}$. Se sugiere el uso de mascarillas específicas N95, FFP2 o superiores ${ }^{21-34}$ o si se dispone del recurso trajes con respirador motorizado purificador de aire (PAPRs) ${ }^{25,27,28,31,34}$, gorro quirúrgico $^{21,24,25,27,31-34}$, protección ocular ${ }^{21,22,24-34}$, bata/ traje quirúrgico impermeable ${ }^{21,22,24-34}$, guantes quirúrgicos ${ }^{21,25,27-30,32,34}$ y cubre calzado ${ }^{24,25,31,33,34}$.
Algunas guías recomiendan, además, el uso de mascarilla quirúrgica superpuesta a la mascarilla específica ${ }^{24}$, o el uso de doble guante quirúrgico o con sistema Elipse $e^{24,26,31,33}$. Todas concuerdan en la importancia de la educación del personal y medidas de esterilización adecuadas que debieran ser implementadas para evitar el contagio al manipular los elementos contaminados tras el procedimiento ${ }^{21-34}$.

\section{Preoperatorio}

Cada caso debiera ser evaluado por un equipo multidisciplinario para contraponer riesgos versus beneficios de realizar la traqueostomía. No existe consenso o guía que ayude al equipo clínico para planificar el momento apropiado para realizar una traqueostomía en el paciente crítico. Múltiples fuentes recomiendan retrasar la intervención, otras en cambio proponen suspender toda traqueostomía electiva debido al alto riesgo de contagio que conlleva, tanto la cirugía como los cuidados posoperatorios $^{9,15}$. No se ha demostrado asociación entre traqueostomía precoz y reducción de mortalidad en estudios retrospectivos y clínicos randomizados de pacientes en ventilación mecánica ${ }^{35,36}$. Según datos del reporte del Intensive Care National Audit \& Research Centre con respecto a pacientes con COVID-19 en cuidado crítico, una gran proporción de pacientes admitidos en UCI con requerimientos de ventilación mecánica fueron extubados con éxito entre los 5 y 10 días, aquellos que requirieron ventilación mecánica (VM) más prolongada se acompañan de un pobre pronóstico general, por lo que se recomienda evaluar caso a caso ${ }^{37}$. Las indicaciones clínicas requeridas para necesitar traqueostomía han sido principalmente obstrucción de vía aérea y falla de intubación primaria en el contexto de urgencia, considerando en algunos centros también la necesidad de VM prolongada ${ }^{26}$.

De forma unánime, se recomienda conocer el estado de positividad de COVID-19 del paciente previa traqueostomía ${ }^{21-34}$. Algunas guías refieren que el no conocer el estado por factores locales, se debe manejar a todo paciente desconocido como COVID-19 positivo ${ }^{23,24,26,27,31,32}$. En el caso de conocer el status de positividad, se recomienda posponer el procedimiento hasta disminuir la carga viral, de 2 a 3 semanas o confirmar con test negativo $23,26-28$. 


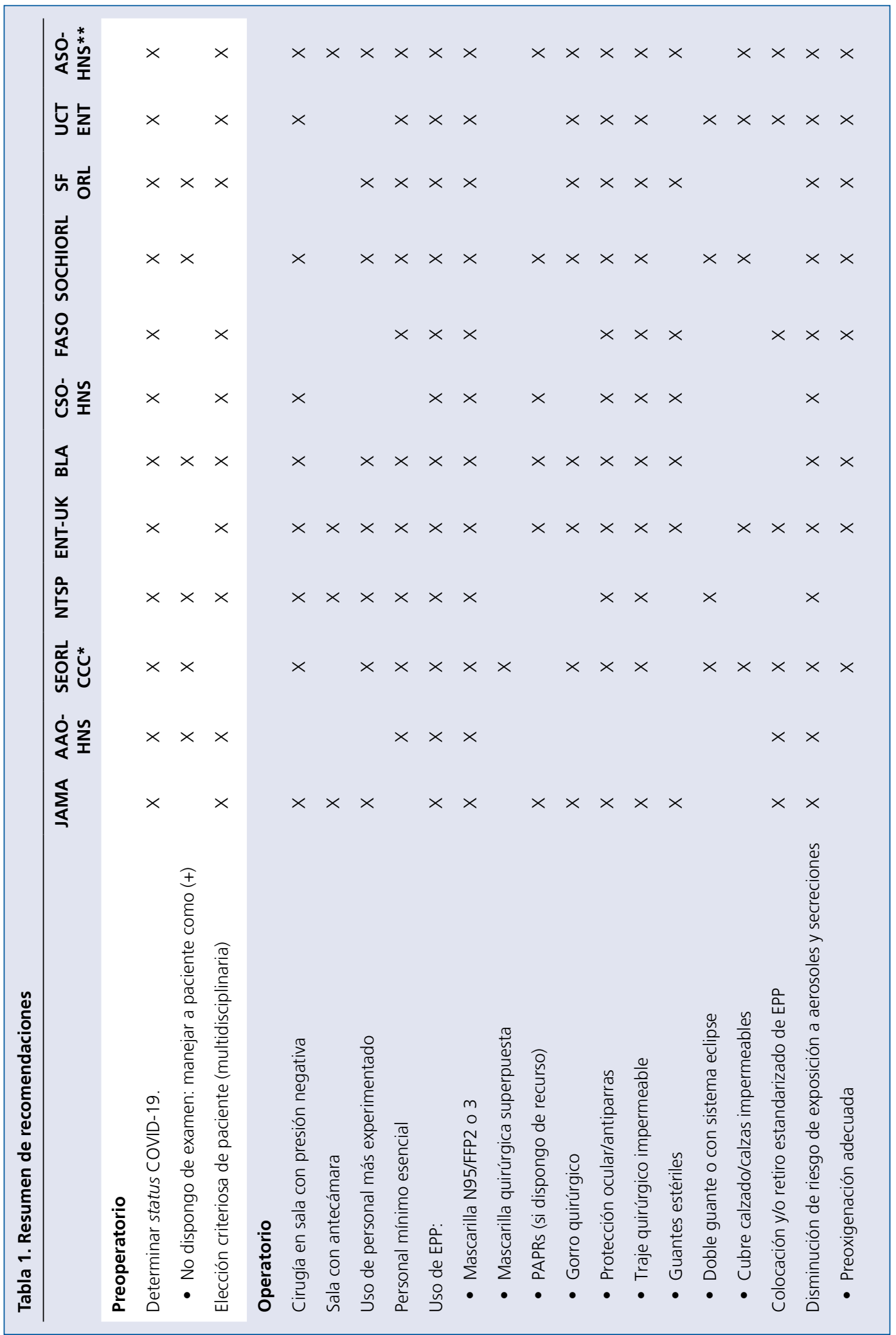




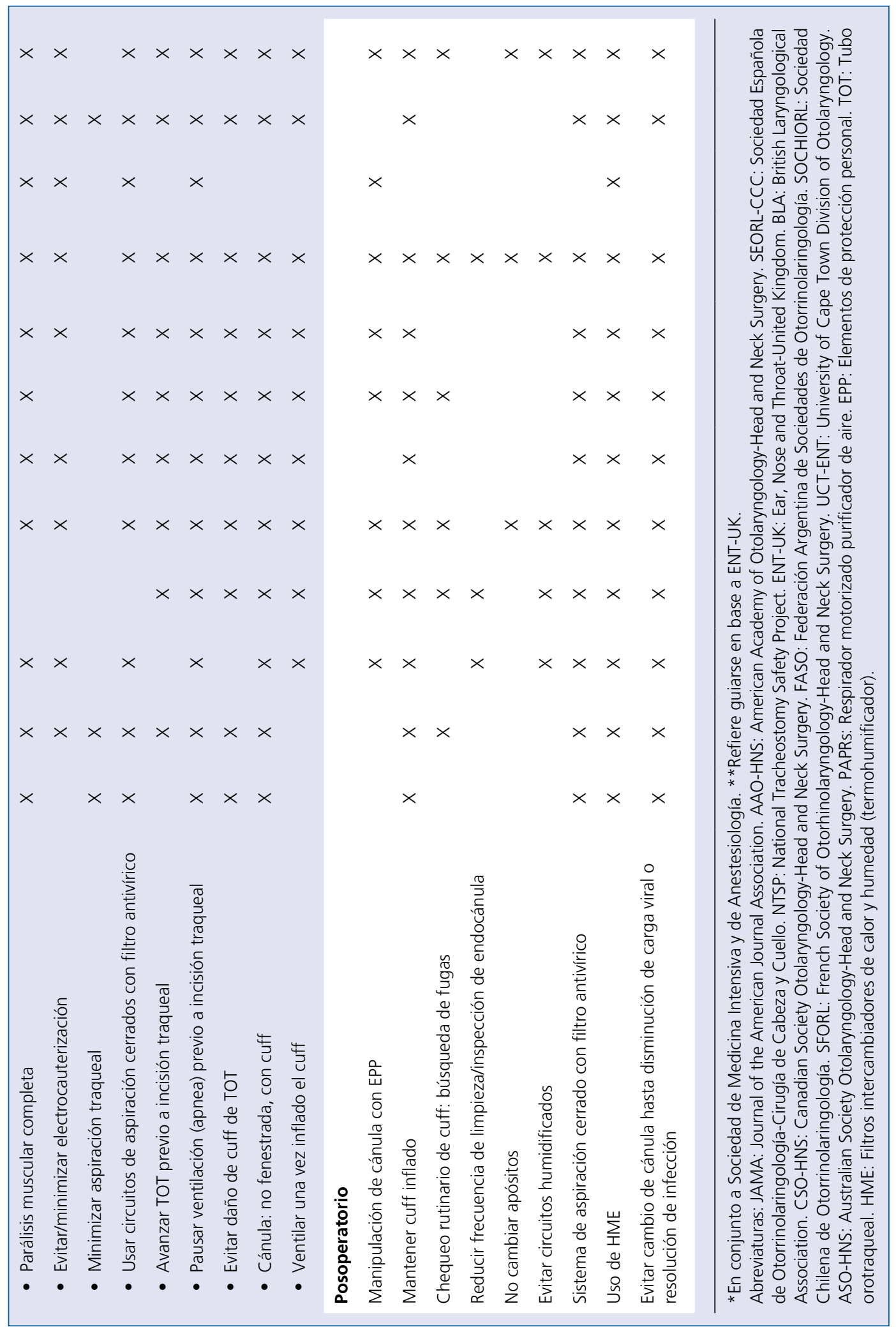




\section{Técnica quirúrgica}

La mayoría de las recomendaciones determinan preferencia sobre un tipo de técnica en particular. Al momento de revisar registros de centros hospitalarios en manejo del brote de SARS de 2002, se describió una mayor manipulación de la vía aérea en la técnica percutánea dado el uso de broncoscopio y dilataciones seriadas $^{22}$. La guía Canadiense ${ }^{28}$ y la Sociedad Británica de Laringología recomiendan la técnica abierta, manifestando que la técnica dependerá del centro y profesionales ${ }^{27}$. Se recomienda utilizar la técnica disponible según los recursos, y grado de expertiz y comodidad del equipo médico para disminuir el tiempo quirúrgico, complicaciones y riesgos asocia$\operatorname{dos}^{21-27,28-34}$.

\section{Manejo operatorio}

Independiente si el procedimiento se realiza en la UCI o pabellón, se recomienda contar con un sistema de presión negativa ${ }^{21,24-28,31,33,34}$. La principal ventaja de realizar el procedimiento en la cama del paciente es evitar traslados innecesarios $^{21,22,32}$. En caso de realizar el procedimiento en la UCI se sugiere contar con una antecámara para aumentar las barreras y estaciones de descontaminación $n^{25,26,34}$. La mayoría de las guías recomiendan la presencia

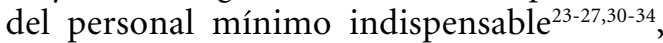
siendo el equipo quirúrgico elegido aquel con mayor experiencia, para disminuir al máximo el tiempo quirúrgico ${ }^{21,24-28,31,32,34}$.

Las recomendaciones intraoperatorias apuntan a disminuir el riesgo de aerosolización de secreciones y exposición del equipo a las mismas $^{21-34}$. Se recomienda preoxigenar adecuadamente al paciente ${ }^{24,25,27,30-34}$, con el objetivo de generar apneas sin afectar la oxigenación. Se sugiere una parálisis completa para evitar el reflejo tusígeno ${ }^{21,23-25,27-34}$. Se recomienda evitar sistemas de corte y coagulación eléctrico por el riesgo de dispersión de micropartículas aéreas ${ }^{23-25,27,30-34}$. También, minimizar el uso de aspiración traqueal directa, o en caso de utilizarlo, que sea en un sistema cerrado con filtro antivírico ${ }^{21-25,27-34}$. Antes de realizar la incisión, la mayoría recomienda avanzar el tubo endotraqueal hasta quedar el cuff por debajo de la zona de acceso a la tráquea, y asegurarse que no existan fugas en el circuito ventilatorio $^{23,25-31,33,34}$. De forma casi unánime se recomienda pausar el sistema de ventilación $\mathrm{y}$ mantener al paciente en apnea justo antes y durante la incisión de la tráquea ${ }^{21-34}$, poniendo especial cuidado en no dañar el cuff del tubo ${ }^{21-}$ 23,25-31,33,34. Posteriormente, se debe instalar una cánula no fenestrada con cuff ${ }^{1-31,33,34}$. Sólo una vez inflado el cuff, se debe reiniciar la ventilación, comprobando la presencia en vía aérea por capnografía ${ }^{24-31,33,34}$. Al finalizar el procedimiento, se debe fijar la cánula. Posteriormente, se deben retirar los EPP y quirúrgicos de forma estandarizada ${ }^{21-25,30,31,33,34}$. A lo largo de todo el procedimiento se recomienda que, ante cualquier momento de desconexión del sistema de ventilación, considerar pausar y mantener en apnea hasta reconectar adecuadamente ${ }^{26}$.

\section{Posoperatorio}

Algunas guías abordan recomendaciones detalladas del manejo posoperatorio del paciente traqueostomizado ${ }^{23-26,29,31,34}$. Se sugiere mantener medidas extremas de seguridad para traslados, minimizar o evitarlos, y en caso de realizarse, todo personal involucrado debe contar con $\mathrm{EPP}^{24-26,29-32,34}$. En la unidad del paciente, se recomienda designar un miembro del equipo de salud dedicado a sostener la cánula y tubo conectado a la traqueostomía cada vez que el paciente se cambie de posición ${ }^{25,31,34}$. Otras medidas recomendadas son: evitar uso de circuitos humidificados ${ }^{24-26,31,34}$, preferir filtros intercambiadores de calor y humedad ${ }^{21-34}$, uso exclusivo de circuitos cerrados de aspiración ${ }^{21,23-31,33,34}$, verificación periódica de presión de cuff en búsqueda de fugas ${ }^{23,25,26,29,31,34}$, mantener cuff inflado ${ }^{21-32,34}$ y mantener apósitos y gasas peritraqueostoma ${ }^{25,31}$.

Se recomienda postergar el primer cambio de cánula el mayor tiempo posible $e^{21,23-31,33,34}$. Las únicas guías que precisan el tiempo para el cambio de cánula son la de la Sociedad Española ${ }^{24}$, del Reino Unido ${ }^{25-27}$ y Sudáfrica ${ }^{33}$, que proponen su cambio entre la primera y tercera semana postraqueostomía ${ }^{24-27,33}$. Otras plantean realizar pruebas moleculares hasta lograr la negativización de muestras previo al cambio de la cánula ${ }^{21,23-25,30-31,34}$. Durante el cambio de la cánula se recomienda pausar la ventilación antes de desinflar el cuff, insertar la nueva cánula e inmediatamente inflar nuevamente el cuff y reconectar el circuito ${ }^{27,31}$. Los cambios subsiguientes se deben postergar lo 
máximo posible, proponiendo intervalos de 30 días $^{27}$. Se debe mantener el uso de cánula con cuff, no fenestrada hasta confirmar la negativización de COVID-19 y considerar el plan de decanulación solo en pacientes con confirmación de negativización de COVID-19 y ser realizado en sector hospitalario libre de coronavirus $^{27}$.

\section{Traqueostomía de urgencia}

Existe el escenario donde el paciente pueda presentarse en el Servicio de Urgencia por insuficiencia respiratoria aguda, con necesidad de manejo inmediato de la vía aérea. Dado que, en este contexto, donde se presentará en su mayoría con síntomas respiratorios graves, sin tiempo para realizar exámenes diagnósticos, se debe manejar a todo paciente como COVID-19 positivo. Algunas guías mencionan la realización de cricotiroidotomía ${ }^{24}$, pero en su mayoría como manejo de salvataje, en caso de fallar la intubación, la traqueostomía de urgencia o no contar con personal entrenado para dichos procedimientos.

\section{Discusión}

La actual pandemia por el SARS-CoV-2 es un suceso dinámico y desafiante en la atención de pacientes para todo el personal sanitario. Dentro de las grandes complicaciones observadas en esta pandemia, destaca su elevada contagiosidad $^{5}$ y capacidad para colapsar la salud pública a lo largo del mundo ${ }^{38}$, lo cual puede conducir a tomar complejas decisiones en el tratamiento de los pacientes y utilización de recursos.

Entre las sociedades y guías consultadas, algunas recomendaciones generales son compartidas por todas ellas, conocer el estatus infeccioso del paciente previo a la realización de la traqueostomía, el uso de EPP y el disminuir el riesgo de exposición a aerosoles y secreciones no presentan discusión. Lo anterior se corresponde con la evidencia publicada hasta el momento donde, a modo de ejemplo, se destaca que el uso adecuado de EPP, junto a una correcta colocación y retiro de tales elementos es clave, resalta una serie de casos en Wuhan que describió que ninguno de los 41 trabajadores de la salud equipados y entrenados con EPP, expuestos a pacientes COVID-19, desarrolló la infección posteriormente ${ }^{14}$.

El manejo del acto quirúrgico electivo inicia con el plan preoperatorio, con la discusión por medio de un equipo multidisciplinario de la necesidad o no de realizar la traqueostomía. Con respecto al momento de cuando realizar el procedimiento, las guías difieren entre realizar un enfrentamiento más conservador o agresivo (precoz). Las guías británicas ${ }^{25-27}$, americanas $^{21-23}$ y sudafricanas ${ }^{33}$ proponen una postura conservadora, intentando evitar el procedimiento en pacientes COVID-19 positivos a menos que sea estrictamente necesario, e idealmente esperar a la negativización por PCR o 14 días posterior al inicio de ventilación mecánica. Por otro lado, el consenso de la Sociedad Francesa sugiere realizar traqueostomía precoz con el objetivo de optimizar el destete a ventilación mecánica, generando mayor disponibilidad en Unidades de Cuidado Intensivo (UCI) ${ }^{32}$. La guía española es cauta en esperar 14 días en pacientes positivos, pero señala que de todas maneras se debe considerar la traqueostomía precoz para optimizar recursos de unidades intensivas, y para pacientes estables con baja demanda de oxígeno en que se prevee ventilación mecánica prolongada ${ }^{24}$. Si bien se ha descrito persistencia de positividad del examen de PCR en muestras del tracto respiratorio bajo hasta los 39 días, los estudios apuntan a un pequeño riesgo residual de infectividad más allá de los 10 días desde el inicio de los síntomas ${ }^{39}$.

Es fundamental contar con todos los EPP necesarios para intervenir a un paciente COVID-19 positivo o de estatus desconocido, tomando consideraciones en el lugar físico a realizar y el equipo quirúrgico. En cuanto a lo operacional, se recomiendan una serie de medidas que apuntan a disminuir el riesgo de exposición a aerosoles y secreciones. Si bien no todas las guías las detallan, es igualmente importante contar con medidas de manejo y cuidado del paciente usuario de traqueostomía durante toda su estadía hospitalaria.

Pese a todas las recomendaciones, en todo el mundo los trabajadores de la salud se han visto afectados por esta enfermedad, llegando incluso a la muerte, lo que genera preocupación y esfuerzos por mantener la seguridad y salud del personal sanitario ${ }^{14,33}$. Según datos reportados en Wuhan, el 3,8\% del total de infectados fueron miembros del personal 
de salud, de los cuales 14,8\% manifestó una enfermedad severa ${ }^{40}$. En Italia, un $15 \%$ del personal de salud resultó afectado ${ }^{41}$. Si bien en el enfrentamiento de la pandemia existe alta concordancia por parte de las sociedades científicas, muchas de estas recomendaciones se generan a raíz de opiniones y sugerencias de expertos en el tema, pero son pocos los estudios que se han logrado realizar para entregar recomendaciones con fuerte nivel de evidencia.

Villares y colaboradores describen una cohorte de 1.890 pacientes COVID-19 en condición crítica en los que se realizó traqueostomía dentro de 120 hospitales en España, equivalente al $16,4 \%$ del total de pacientes en UCI en ventilación mecánica. Destaca que en su mayoría fueron procedimientos programados $(99,8 \%)$, de técnica quirúrgica abierta $(81,3 \%)$, con una mediana de tiempo de 12 días posintubación (rango de 4-42 días). En la gran mayoría se realizó la cirugía en la unidad del paciente en UCI, con una baja tasa de complicaciones. El personal de salud dispuso de EPP, antiparras, mascarillas N95/ FFP2 con mascarilla quirúrgica superpuesta, doble guante quirúrgico y escudo facial, en 2 hospitales existía disponibilidad de PAPRs. Se realizó seguimiento con respecto a los resultados de 1.616 pacientes hasta el momento de la publicación del artículo, dentro de los cuales en $52,1 \%$ se logró el destete y un $23,7 \%$ murió producto de la enfermedad, el porcentaje restante persistía en ventilación mecánica. En aquellos en que se logró el destete, la decanulación fue exitosa en un $81 \%$. No se indicó la tasa de infección dentro del personal de salud participante en los procedimientos ${ }^{42}$.

Las principales limitaciones de esta revisión se deben al conocimiento en constante evolución respecto a la pandemia $S A R S-C o V-2$ y la ausencia de estudios prospectivos a gran escala, por lo que es de suponer que las conclusiones y recomendaciones aquí entregadas, así como también las de las distintas identidades enunciadas previamente puedan presentar cambios a futuro.

\section{Conclusiones}

Esta revisión resume las principales recomendaciones nacionales e internacionales actualizadas a la fecha para la realización y cuidado de un paciente con traqueostomía, procedimiento ampliamente realizado en estas circunstancias. Algunas recomendaciones se basan fundamentalmente en la experiencia previa del SARS en 2002, otras en pequeñas series de casos en China, país de inicio de la pandemia COVID-19. Muchas de las recomendaciones son compartidas entre las distintas entidades, desde determinar previo al procedimiento el status infeccioso del paciente hasta ciertos tópicos del manejo operatorio, contribuyendo esto al aprendizaje y a unificar las líneas de acción en el manejo de estos pacientes. Por otra parte, existen también diferencias, que son por lo general menores y relacionadas a la implementación de los EPP e insumos médicos, que, dentro de distintos factores, pueden estar sujetas a la disponibilidad local y costos asociados. De momento las ventajas de la traqueostomía impresionan ser su apoyo en el destete de ventilación mecánica, contribuyendo a la descongestión de unidades intensivas. Sin embargo, sus indicaciones, decisión del timing quirúrgico e impacto en supervivencia carecen de mayores datos prospectivos para generar recomendaciones categóricas.

\section{Bibliografía}

1. Zhu N, Zhang D, Wang W, et al. A novel coronavirus from patients with pneumonia in China, 2019. N Engl J Med. 2020;382(8):727-733. doi: 10.1056/ NEJMoa2001017.

2. Guan WJ, Ni ZY, Hu Y, et al. Clinical characteristics of coronavirus disease 2019 in China. N Engl J Med. 2020;382(18):1708-1720. doi: 10.1056/ NEJMoa2002032.

3. Singhal T. A review of coronavirus disease-2019 (COVID-19). Indian J Pediatr. 2020;87(4):281-286. doi: 10.1007/s12098-020-03263-6.

4. Munster VJ, Koopmans M, van Doremalen N, van Riel D, de Wit E. A novel coronavirus emerging in China-key questions for impact assessment. $N$ Engl J Med. 2020;382(8):692-694. doi: 10.1056/ NEJMp2000929.

5. Salas-Asencios R, Iannacone-Oliver J, GuillénOneeglio A, et al. Coronavirus COVID-19: Conociendo al causante de la Pandemia. The Biologist. 2020; 18:9-27. doi: 10.24039/rtb2020181442.

6. World Health Organization. WHO Coronavirus disease 2019 (COVID-19) Dashboard. Disponible en: https://covid19.who.int/. Consultado el 30 de 
septiembre de 2020.

7. MINSAL. Plan de acción Coronavirus COVID-19. Reporte Diario COVID-19 30-09-2020.

Disponible en: https://www.minsal.cl/wp-content/ uploads/2020/09/CP-REPORTE-COVID-19Mi\%C3\%A9rcoles-30092020.pdf. Consultado el 30 de septiembre de 2020.

8. Jin YH, Cai L, Cheng ZS, et al. A rapid advice guideline for the diagnosis and treatment of 2019 novel coronavirus (2019-nCoV) infected pneumonia (standard version). Mil Med Res. 2020;7(1):4. doi: 10.1186/s40779-020-0233-6.

9. van Doremalen N, Bushmaker T, Morris DH, et al. Aerosol and surface stability of SARS-CoV-2 as compared with SARS-CoV-1. N Engl J Med. 2020; 382:1564-1567. doi: 10.1056/NEJMc2004973.

10. Meo SA, Alhowikan AM, Al-Khlaiwi T, et al. Novel coronavirus 2019-nCoV: prevalence, biological and clinical characteristics comparison with SARS-CoV and MERS-CoV. Eur Rev Med Pharmacol Sci. 2020; 24 (4):2012-2019. doi: 10.26355/ eurrev_202002_20379.

11. Lake MA. What we know so far: COVID-19 current clinical knowledge and research. Clin Med (Lond). 2020;20(2):124-127. doi: 10.7861/ clinmed.2019-coron.

12. Adhikari SP, Meng S, Wu YJ, et al. Epidemiology, causes, clinical manifestation and diagnosis, prevention and control of coronavirus disease (COVID-19) during the early outbreak period: a scoping review. Infect Dis Poverty. 2020;9(1):29. doi: 10.1186/s40249-020-00646-x.

13. Meng L, Hua F, Bian Z. Coronavirus Disease 2019 (COVID-19): Emerging and Future Challenges for Dental and Oral Medicine. J Dent Res. 2020;99(5):481-487. doi: 10.1177/0022034520914246.

14. Ran L, Chen X, Wang Y, Wu W, Zhang L, Tan X. Risk Factors of Healthcare Workers with Corona Virus Disease 2019: A Retrospective Cohort Study in a Designated Hospital of Wuhan in China. Clin Infect Dis. 2020; ciaa287. doi: 10.1093/cid/ciaa287.

15. Tay JK, Khoo ML, Loh WS. Surgical Considerations for Tracheostomy During the COVID-19 Pandemic: Lessons Learned From the Severe Acute Respiratory Syndrome Outbreak. JAMA Otolaryngol Head Neck Surg. 2020;146(6):517-518. doi: 10.1001/ jamaoto.2020.0764.

16. Patel ZM, Fernández-Miranda J, Hwang PH, et al. Precautions for endoscopic transnasal skull base surgery during the COVID-19 pandemic. Neurosurgery. 2020; nyaa125. doi: 10.1093/neuros/ nyaa125.

17. Vargas M, Servillo G. Improving staff safety during tracheostomy in COVID-19 patients. Head Neck. 2020;1-2. doi: https://doi.org/10.1002/ hed.26163.
18. Kwan A, Fok WG, Law KI, Lam SH. Tracheostomy in a patient with severe acute respiratory syndrome. $\mathrm{Br} \mathrm{J}$ Anaesth. 2004;92(2):280-2. doi: 10.1093/bja/aeh035.

19. Chee VW, Khoo ML, Lee SF, Lai YC, Chin NM. Infection control measures for operative procedures in severe acute respiratory syndrome-related patients. Anesthesiology. 2004;100(6):1394-8. doi: 10.1097/00000542-200406000-00010.

20. Tien HC, Jogeklar A, Cooper AB, \& Brenneman F. Elective and emergency surgery in patients with severe acute respiratory syndrome (SARS). Can J Surg. 2005;48(1): 71-74.

21. Givi B, Schiff BA, Chinn SB, et al. Safety Recommendations for Evaluation and Surgery of the Head and Neck During the COVID-19 Pandemic. JAMA Otolaryngol Head Neck Surg. 2020;146(6):579-584. doi: 10.1001/ jamaoto.2020.0780.

22. Chan JYK, Wong EWY, Lam W. Practical Aspects of Otolaryngologic Clinical Services During the 2019 Novel Coronavirus Epidemic: An Experience in Hong Kong. JAMA Otolaryngol Head Neck Surg. 2020;146(6):519-520. doi: 10.1001/ jamaoto.2020.0488.

23. Parker N, Schiff B, Fritz M, et al. Tracheotomy Recommendations During the COVID-19 Pandemic. Airway and Swallowing Committee of the American Academy of Otolaryngology-Head and Neck Surgery. Disponible en: https://www.entnet.org/content/ tracheotomy-recommendations-during-covid-19pandemic. Consultado el 10 de abril de 2020.

24. Villalonga Vadell R, Martín Delgado MC, AvilésJurado FX, et al. Consensus Document of the Spanish Society of Intensive and Critical Care Medicine and Coronary Units (SEMICYUC), the Spanish Society of Otorhinolaryngology and Head and Neck Surgery (SEORL-CCC) and the Spanish Society of Anesthesiology and Resuscitation (SEDAR) on Tracheotomy in Patients with COVID-19 Infection [published online ahead of print, 2020 Jun 10]. Documento de consenso de la Sociedad Española de Medicina Intensiva, Crítica, y Unidades Coronarias (SEMICYUC), la Sociedad Española de Otorrinolaringología y Cirugía de Cabeza y Cuello (SEORL-CCC) y la Sociedad Española de Anestesiología y Reanimación (SEDAR) sobre la traqueotomía en pacientes con COVID-19 [published online ahead of print, 2020 Jun 10]. Rev Esp Anestesiol Reanim. 2020;S0034-9356(20)30116-X. doi: 10.1016/j. redar.2020.05.001.

25. ENTUK. Tracheostomy guidance during the COVID-19 Pandemic - ENT-UK. Disponible en: https://www.entuk.org/tracheostomy-guidanceduring-covid-19-pandemic?page $=1$. Consultado el 6 de mayo de 2020.

26. National Tracheostomy Safety Project. NTSP considerations for tracheostomy in the COVID-19 
outbreak. Disponible en: http://www.tracheostomy. org.uk/storage/files/NTSP\%20COVID_19\%20 tracheostomy\%20guidance\%2031_3_20.pdf. Consultado el 10 de abril de 2020

27. British Laryngological Association. COVID-19 Tracheostomy Guideline. Disponible en: https://www. britishlaryngological.org/sites/default/files/BLA\%20 Tracheostomy\%20guideline \%20-BLA\%20April\%20 2020\%20FINAL.pdf. Consultado el 20 de septiembre de 2020.

28. Engels PT, Weitzel E, Witterick IJ, et al. Recommendations from the CSO-HNS Taskforce on Performance of Tracheotomy During the COVID-19 Pandemic. J Otolaryngol Head Neck Surg. 2020;49(1):23. Published 2020 Apr 27. doi: 10.1186/ s40463-020-00414-9. Disponible en: https://www. entcanada.org/wp-content/uploads/COVID-19Guidelines-CSOHNS-Task-Force-Mar-23-2020.pdf. Consultado el 30 de abril de 2020.

29. Sommer DD, Witterick IJ, Jeffery C, Chan Y, Brown T, Côte D, et al. Recommendations from the CSOHNS taskforce on post-operative care following tracheotomy during the COVID-19 pandemic. Disponible en: https://www.entcanada.org/wpcontent/uploads/Recommendations-CSO-HNSTaskforce-V2.0-Sommer-04-20-20.pdf. Consultado el 25 de agosto de 2020.

30. Acosta L, Legris A, Cajelli AL, et al. Protocolo interno COVID-19 para consultas y prácticas del servicio de Otorrinolaringología, Hospital Italiano de Buenos Aires. Disponible en: http://faso.org.ar/imagenes/ COVID/protocolo_italiano-17-4.pdf. Consultado el 20 de abril de 2020.

31. Sociedad Chilena de Otorrinolaringología. Medicina y Cirugía de Cabeza y Cuello. Recomendaciones para realización de traqueostomías en paciente con covid-19 (+) o sospechoso. Disponible en: https://sochiorl.cl/web/imgnuevas/videos_covid/ recomendaciones_traqueostomia.pdf. Consultado el 6 de mayo de 2020.

32. Schultz P, Morvan JB, Fakhry N, et al. French consensus regarding precautions during tracheostomy and post-tracheostomy care in the context of COVID-19 pandemic. Eur Ann Otorhinolaryngol Head Neck Dis. 2020;137(3):167-169. doi: 10.1016/j. anorl.2020.04.006.

33. University of Cape Town Division of Otolaryngology. Tracheostomy for COVID-19 positive cases.
Disponible en: https://drive.google.com/file/ d/1OMSR7T12tlO9tJ-TqbBmImo9nc63HwCt/view. Consultado el 20 de septiembre de 2020.

34. ASOHNS. Guidance for ENT surgeons during the COVID-19 pandemic. Disponible en: http:// www.asohns.org.au/CMS/Uploads/COVID_19_ Tracheostomy_guidance_18_March_2020.pdf. Consultado el 10 de abril de 2020.

35. Young D, Harrison DA, Cuthbertson BH, Rowan K, TracMan Collaborators. Effect of early vs late tracheostomy placement on survival in patients receiving mechanical ventilation: the TracMan randomized trial. JAMA. 2013;309(20):2121-9. doi: 10.1001/jama.2013.5154.

36. Choi KW, Chau TN, Tsang O, et al. Outcomes and prognostic factors in 267 patients with severe acute respiratory syndrome in Hong Kong. Ann Intern Med. 2003;139(9):715-23. doi: 10.7326/0003-4819-139-9200311040-00005.

37. Intensive Care National Audit \& Research Centre (2020) ICNARC report on COVID-19 in critical care. 24 April 2020. Disponible en: https://www.icnarc.org/ Our-Audit/Audits/Cmp/Reports. Consultado el $28 \mathrm{de}$ abril de 2020.

38. Hartley DM, Perencevich EN. Public Health Interventions for COVID-19: Emerging Evidence and Implications for an Evolving Public Health Crisis. JAMA. Disponible en: https://jamanetwork. com/journals/jama/fullarticle/2764656. Consultado el 24 de abril de 2020.

39. McGrath BA, Brenner MJ, Warrillow SJ, et al. Tracheostomy in the COVID-19 era: global and multidisciplinary guidance. Lancet Respir Med. 2020;8(7):717-725. doi: 10.1016/S22132600(20)30230-7.

40. Wang J, Zhou M, Liu F. Exploring the reasons for healthcare workers infected with novel coronavirus disease 2019 (COVID-19) in China. J Hosp Infect. 2020. doi: 10.1016/j.jhin.2020.03.002.

41. Remuzzi A, Remuzzi G. COVID-19 and Italy: what next? Lancet. 2020;395(10231):1225-1228. doi: 10.1016/S0140-6736(20)30627-9.

42. Martin-Villares C, Pérez Molina-Ramirez C, BartoloméBenito M, Bernal-Sprekelsen M; COVID ORL ESP Collaborative Group ${ }^{\star}$. Outcome of 1890 tracheostomies for critical COVID-19 patients: a national cohort study in Spain. Eur Arch Otorhinolaryngol. 2020;1-8. doi: 10.1007/s00405-020-06220-3. 